TAIWANESE JOURNAL OF MATHEMATICS

Vol. 17, No. 1, pp. 133-142, February 2013

DOI: $10.11650 /$ tjm.17.2013.1803

This paper is available online at http://journal.taiwanmathsoc.org.tw

\title{
ON PRIME SUBMODULES AND PRIMARY DECOMPOSITIONS IN TWO-GENERATED FREE MODULES
}

\author{
Seçil Çeken and Mustafa Alkan
}

\begin{abstract}
In this paper, we consider the free $R$-module $R \oplus R$, where $R$ is an arbitrary commutative ring with identity. We give a full characterization for prime submodules of $R \oplus R$ and a useful primeness test for a finitely generated submodule of $R \oplus R$. We study the existence of primary decomposition of a submodule of $R \oplus R$ and characterize the minimal primary decomposition. As applications of our results, we give some examples of primary decompositions in $R \oplus R$.
\end{abstract}

\section{INTRODUCTION}

Throughout this paper, all rings are commutative with identity and all modules are unitary.

Let $R$ be a ring and $M$ be an $R$-module. For any submodule $N$ of $M$ we set $(N: M)=\{r \in R: r M \subseteq N\}$. A proper submodule $N$ of $M$ is called a $P$ prime submodule if $r m \in N$ for $r \in R$ and $m \in M$ implies that either $m \in N$ or $r \in P=(N: M)$. It is well-known that a proper submodule $N$ of $M$ is prime if and only if $P$ is a prime ideal of $R$ and $M / N$ is torsion-free as an $R / P$-module.

A proper submodule $Q$ of $M$ is called a primary submodule provided that for any $s \in R$ and $m \in M, s m \in Q$ implies that $m \in Q$ or $s^{n} \in(Q: M)$ for some positive integer $n$. Let $Q$ be a primary submodule of $M$, then the radical of the ideal $(Q: M)$ is a prime ideal of $R$. If $P=\sqrt{(Q: M)}$, then $Q$ is called a $P$-primary submodule of $M$.

A submodule $N$ of $M$ has a primary decomposition if $N=Q_{1} \cap \ldots \cap Q_{k}$ with each $Q_{i}$ a $P_{i}$-primary submodule of $M$ for some prime ideal $P_{i}$. If no $Q_{i}$ contains $Q_{1} \cap \ldots \cap Q_{i-1} \cap Q_{i+1} \cap \ldots \cap Q_{k}$ and the ideals $P_{1}, \ldots, P_{k}$ are all distinct, then the primary decomposition is said to be minimal and the set $\operatorname{Ass}(N)=\left\{P_{1}, \ldots, P_{k}\right\}$ is said to be the set of associated prime ideals of $N$.

Received April 5, 2012, accepted June 7, 2012.

Communicated by Bernd Ulrich.

2010 Mathematics Subject Classification: 13A99, 13C10, 13 C99.

Key words and phrases: Prime submodule, Primary submodule, Primary decomposition. 
Let $I$ be an ideal of $R$ such that $I$ has a primary decomposition. It is well-known that the minimal members of $A s s(I)$ are precisely the minimal prime ideals of $I$. These prime ideals are called the minimal primes of $I$. The remaining associated primes of $I$, that is, the associated primes of $I$ which are not minimal, are called the embedded primes of $I$.

Prime submodules and primary decompositions of submodules of a module over a commutative ring have been studied by many authors (see, [6], [7], and [11]). In [10], Tıraş and Harmancı gave some characterizations of prime and primary submodules of $R \oplus R$, where $R$ is a PID (Principal Ideal Domain). Moreover, these submodules of finitely generated free modules over a PID were studied in [3], [4], [5] and [1]. PusatYllmaz in [9] also studied prime submodules of finitely generated free modules over arbitrary commutative domains.

In this paper, we completely determine prime submodules of $R \oplus R$ for an arbitrary commutative ring $R$, and we generalize some known results in [10] and [9]. We also study the existence of the primary decomposition of a submodule of $R \oplus R$, and characterize the minimal primary decomposition. As applications of our results, we give some examples of primary decompositions in $R \oplus R$, where $R$ is not a PID.

\section{Prime Submodules and Primary Decompositions}

In the rest of this paper we fix the following notations: Let $R$ be a commutative ring with identity and $F=R \oplus R$. We use $N$ to be a non-zero submodule of $F$ generated by the set $\left\{\left(a_{i}, b_{i}\right) \in F: i \in \Lambda\right\}$ and $\mathcal{L}=\sum_{i, j \in \Lambda} R \Delta_{i j}$ where $\Delta_{i j}=a_{i} b_{j}-b_{i} a_{j}$ for $i, j \in \Lambda$.

The following Lemma can be found in [2]. But we give its proof for completeness.

Lemma 2.1. Let $F$ and $N$ be as above. Then $\mathcal{L} \subseteq(N: F) \subseteq \sqrt{\mathcal{L}}$.

Proof. For all $i, j \in \Lambda$, we have

$$
\begin{aligned}
& \Delta_{i j}(1,0)=\left(a_{i}, b_{i}\right) b_{j}-\left(a_{j}, b_{j}\right) b_{i} \in N \\
& \Delta_{i j}(0,1)=\left(a_{j}, b_{j}\right) a_{i}-\left(a_{i}, b_{i}\right) a_{j} \in N
\end{aligned}
$$

and so $\sum_{i, j \in \Lambda} R \Delta_{i j} \subseteq(N: F)$.

Let $x \in(N: F)$. Then there exists a finite subset $\Upsilon$ of $\Lambda$ such that $x(1,0)=$ $\sum_{i \in \Upsilon} t_{i}\left(a_{i}, b_{i}\right)$ and $x(0,1)=\sum_{i \in \Upsilon} k_{i}\left(a_{i}, b_{i}\right)$, where $t_{i}, k_{i} \in R$ for all $i \in \Upsilon$. Then $x=\sum_{i \in \Upsilon} t_{i} a_{i}, x=\sum_{i \in \Upsilon} k_{i} b_{i}, 0=\sum_{i \in \Upsilon} t_{i} b_{i}$ and $0=\sum_{i \in \Upsilon} k_{i} a_{i}$. Thus we have

$$
\begin{aligned}
x^{2} & =\sum_{i \in \Upsilon} t_{i} k_{i} a_{i} b_{i}+\sum_{i \in \Upsilon}\left(\sum_{j \in \Upsilon, i \neq j} t_{i} a_{i} k_{j} b_{j}\right) \\
0 & =\sum_{i \in \Upsilon} t_{i} k_{i} a_{i} b_{i}+\sum_{i \in \Upsilon}\left(\sum_{j \in \Upsilon, i \neq j} t_{i} b_{i} k_{j} a_{j}\right) .
\end{aligned}
$$

Therefore, $x^{2}=\sum_{i \in \Upsilon}\left(\sum_{j \in \Upsilon, i \neq j} t_{i} k_{j} \Delta_{i j}\right) \in \sum_{i, j \in \Upsilon} R \Delta_{i j}$ and so $x \in \sqrt{\mathcal{L}}$. 
Theorem 2.2. Let $N$ be a submodule of $F$ with $(N: F)=P$.

(a) If $P$ is a prime ideal of $R$ and $a_{i}, b_{i} \in P$ for all $i \in \Lambda$, then $N=P \oplus P$ and $N$ is a prime submodule.

(b) If $P$ is a maximal ideal of $R, a_{i} \notin P$ for some $i \in \Lambda$ and $b_{j} \notin P$ for some $j \in \Lambda$, then $N=\left(a_{i} b_{j}\right) N+P F$ and $N$ is a prime submodule.

(c) If $\left\{a_{i}: i \in \Lambda\right\} \cup\left\{b_{i}: i \in \Lambda\right\} \nsubseteq P$ and $N$ is a prime submodule, then $N=$ $\left\{(m, n) \in F: m b_{i}-n a_{i} \in P\right.$ for all $\left.i \in \Lambda\right\}$. In particular,

(i) If $a_{i} \in P$ for all $i \in \Lambda$ and $b_{j} \notin P$ for some $j \in \Lambda$ (resp. $b_{i} \in P$ for all $i \in \Lambda$ and $a_{j} \notin P$ for some $j \in \Lambda$ ) then $N=P \oplus R$ (resp. $N=R \oplus P$ ).

(ii) If $a_{i} \notin P$ and $b_{i} \in P$ for some $i \in \Lambda$ (resp. $b_{i} \notin P$ and $a_{i} \in P$ for some $i \in \Lambda)$ then $N=R \oplus P($ resp. $N=P \oplus R)$.

Proof.

(a) It is clear that $P F$ is a prime submodule of $F$ contained in $N$. If $a_{i}, b_{i} \in P$ for all $i \in \Lambda$ then $P F=P \oplus P$ contains $N$.

(b) Let $P$ be a maximal ideal of $R$ and let $a_{i} \notin P$ for some $i \in \Lambda$ and $b_{j} \notin P$ for some $j \in \Lambda$. Then we get that $\left(a_{i} b_{j}\right) R+P=R$. Let $(x, y) \in N$. Then $x=r a_{i} b_{j}+p_{1}$ and $y=s a_{i} b_{j}+p_{2}$ for some $r, s \in R$ and $p_{1}, p_{2} \in P$. It follows that $(x, y)=a_{i} b_{j}(r, s)+\left(p_{1}, p_{2}\right)$ and then $a_{i} b_{j}(r, s) \in N$ as $\left(p_{1}, p_{2}\right) \in P F \subseteq N$. Since $(N: F)=P$ is a maximal ideal of $R, N$ is a prime submodule of $F$ and hence $(r, s) \in N$. Thus $(x, y) \in\left(a_{i} b_{j}\right) N+P F$ and so $N \subseteq\left(a_{i} b_{j}\right) N+P F$. The other inclusion is clear.

(c) We may assume that $a_{1} \notin P$. Consider the submodule

$$
T_{P}=\left\{(m, n) \in F: m b_{i}-n a_{i} \in P \text { for all } i \in \Lambda\right\} .
$$

By Lemma 2.1, it is clear that $N \subseteq T_{P}$. Let $(m, n) \in T_{P}$. Then there exists a $p \in P$ such that $n a_{1}=m b_{1}+p$ and so

$a_{1}(m, n)=\left(a_{1} m, a_{1} n\right)=\left(a_{1} m, b_{1} m\right)+(0, p)=m\left(a_{1}, b_{1}\right)+(0, p) \in N+P F=$ $N$. Since $a_{1} \notin P$, we get that $(m, n) \in N$ and so $T_{P}=N$.

(i) Let $a_{i} \in P$ for all $i \in \Lambda$ and $b_{j} \notin P$ for some $j \in \Lambda$. It is clear that $N \subseteq P \oplus R$. $N=\left\{(m, n) \in F: m b_{i}-n a_{i} \in P\right.$ for all $\left.i \in \Lambda\right\}$ by $(c)$. Let $(x, y) \in P \oplus R$. Then $x b_{i}-y a_{i} \in P$ for all $i \in \Lambda$ and so $(x, y) \in N$. Thus $N=P \oplus R$.

By using the same argument as above it can be proved that $N=R \oplus P$ if $b_{i} \in P$ for all $i \in \Lambda$ and $a_{j} \notin P$ for some $j \in \Lambda$.

(ii) Let $a_{i} \notin P$ and $b_{i} \in P$ for some $i \in \Lambda$. Let $(x, y) \in N$. Then $x b_{i}-y a_{i} \in P$ and so $N \subseteq R \oplus P$. Since $x b_{j}-y a_{j} \in P$ for all $j \in \Lambda$, we get $x b_{j} \in P$. If $x \in P$ then $N=P \oplus P$. This is a contradiction as $\left(a_{i}, b_{i}\right) \in N-(P \oplus P)$. Therefore $b_{j} \in P$ for all $j \in \Lambda$. Now the result follows from $(i)$. 
By using the same argument as above it can be proved that $N=P \oplus R$ if $b_{i} \notin P$ and $a_{i} \in P$ for some $i \in \Lambda$.

By using Theorem 2.2, we prove the following corollary which is a generalization of [10, Proposition 2.3] with a different proof.

Corollary 2.3. Let $N$ be a prime submodule of $F$. Then

(a) If $(1,0) \in N$ then $N=R \oplus(N: F)$.

(b) If $(0,1) \in N$ then $N=(N: F) \oplus R$.

Proof. $\quad(a)$ Let $(1,0) \in N$. It is clear that $N \neq P \oplus P$. Then $b_{i} \in P$, for all $i \in \Lambda$ by Theorem $2.2(c)$. We get that $N=R \oplus(N: F)$ by Theorem $2.2(c-i)$.

Let $N$ be a $P$-prime submodule of a module $M$. It is said that $N$ has $P$-height $n$ for some non-negative integer $n$, if there exists a chain $K_{n} \subset K_{n-1} \subset \ldots \subset K_{1} \subset K_{0}=N$ of $P$-prime submodules $K_{i}$ of $M$, but no longer such chain.

Proposition 2.4. Let $N$ be a P-prime submodule of $F$. If $N \neq P \oplus P$ then the P-height of $N$ is 1 .

Proof. Since $N \neq P \oplus P$, we have that $a_{i} \notin P$ for some $i \in \Lambda$ or $b_{j} \notin P$ for some $j \in \Lambda$. By Theorem 2.2, $N=\left\{(m, n) \in F: m b_{i}-n a_{i} \in P\right.$ for all $\left.i \in \Lambda\right\}$. Let $K$ be a $P$-prime submodule of $F$ with $K \subseteq N$ and let $\left\{\left(c_{i}, d_{i}\right) \in F: i \in \Omega\right\}$ be a generating set for $K$. If $c_{i}, d_{i} \in P$ for all $i \in \Omega$ then $K=P \oplus P$. Suppose that $c_{k} \notin P$ for some $k \in \Omega$ or $d_{l} \notin P$ for some $l \in \Omega$. Then $K=\left\{(x, y) \in F: x d_{i}-y c_{i} \in P\right.$ for all $i \in \Omega\}$ by Theorem 2.2-(c). Since $\left(c_{i}, d_{i}\right) \in N$ for all $i \in \Omega$ we get that $c_{i} b_{j}-d_{i} a_{j} \in P$ and so $\left(a_{j}, b_{j}\right) \in K$ for all $j \in \Lambda$. Hence $K=N$.

Corollary 2.5. Let $N$ be a P-prime submodule of $F$. If $(R \oplus P) \cap N \neq P F$ (resp. $(P \oplus R) \cap N \neq P F)$, then $N=R \oplus P$ (resp. $P \oplus R$ ).

Proof. $\quad$ Let $N$ be a $P$-prime submodule of $F$. Then $(R \oplus P) \cap N$ is a $P$-prime submodule. By Proposition 2.4, we get that $N=(R \oplus P) \cap N$ and so $P \oplus P \subset N \subseteq$ $R \oplus P$. The $P$-height of $R \oplus P$ is 1 by Proposition 2.4. Thus we have $R \oplus P=N$.

Theorem 2.6. Let $N$ be a submodule of $F$ which doesn't contain $(1,0)$ and $(0,1)$. Let $P=(N: F)$ be a maximal ideal of $R$ and $(a, b) \in N$ with $R a+R b \nsubseteq P$. Then $N=\{(x, y) \in F: a y-b x \in P\}$ and $N$ is a prime submodule of $F$.

Proof. Since $(N: F)=P$ is a maximal ideal of $R, N$ is prime.

Assume that $a \in P$. Since $(a, 0) \in N$, we have $(0, b)=b(0,1) \in N$ and so $b \in P$. Thus $R a+R b \subseteq P$, a contradiction, and we get that $a, b \notin P$. Therefore, there exist $x_{1}, y_{1} \in R$ and $p_{1}, p_{2} \in P$ such that $a x_{1}+p_{1}=1, b y_{1}+p_{2}=1$. Let $K=\{(x, y) \in F: a y-b x \in P\}$. Clearly, $K$ is a $P$-prime submodule of $F$. 
To show the equality $N=K$, take $(c, d) \in N$. Since $(a d-b c, 0)=d(a, b)-$ $b(c, d) \in N$ and $(1,0) \notin N$, we get that $a d-b c \in P$ and so $N \subseteq K$. For the reverse inclusion, take $(c, d) \in K$. Then we get that

$$
(c, d)=\left(b y_{1} c+p_{2} c, a x_{1} d+p_{1} d\right)=\left(b y_{1} c, a x_{1} d\right)+\left(p_{2} c, p_{1} d\right)
$$

Since $\left(p_{2} c, p_{1} d\right) \in P \oplus P$, it is enough to show that $\left(b y_{1} c, a x_{1} d\right) \in N$.

Since $x_{1}(a, b) \in N$, it follows that

$$
\left(a x_{1}, b x_{1}\right)+\left(p_{1}, 0\right)=\left(1, b x_{1}\right)=\left(b y_{1}, b x_{1}\right)+\left(p_{2}, 0\right) \in N
$$

and so $b\left(y_{1}, x_{1}\right) \in N$. Then we have $\left(y_{1}, x_{1}\right) \in N$ as $b \notin P$. On the other hand, there exists $q \in P$ such that $b c=q+a d$. Then we get that

$$
\left(y_{1}(b c), a x_{1} d\right)=\left(y_{1} a d+y_{1} q, a x_{1} d\right)=a d\left(y_{1}, x_{1}\right)+\left(q y_{1}, 0\right) .
$$

Therefore, $\left(b y_{1} c, a x_{1} d\right) \in N$ and so $K=N$.

In [8], Pusat-Yılmaz and Smith defined the submodule $K(N, P)=\{m \in M$ : $c m \in N+P M$ for $c \in R \backslash P\}$ for an $R$-module $M$ and $N \leq M$. Then they showed that $K(N, P)=M$ or $K(N, P)$ is the smallest $P$-prime submodule containing $N$. As a consequence of Theorem 2.6, we obtain the following corollary which characterizes $K(N, P)$ and the structure of a prime submodule of $F$. Corollary 2.7-(2) is a generalization of [10, Theorem 2.7].

Corollary 2.7. (1) Let $N$ be a submodule of $F, P=(N: F)$ be a prime ideal and $(a, b) \in N$ with $R a+R b \nsubseteq P$. If $N_{P}$ doesn't contain $\left(\frac{1}{1}, \frac{0}{1}\right)$ and $\left(\frac{0}{1}, \frac{1}{1}\right)$, then $\{(x, y) \in F: a y-b x \in P\}=K(N, P)$.

(2) Let $N$ be a submodule of $F$ which doesn't contain $(1,0)$ and $(0,1)$. Suppose that $P=(N: F)$ is a prime ideal of $R$ and $(a, b) \in N$ with $R a+R b \nsubseteq P$. Then $N$ is a P-prime submodule of $F$ if and only if $N=\{(x, y) \in F:$ ay $-b x \in P\}$.

Proof. (1) Since $P_{P}=(N: F)_{P}=\left(N_{P}: F_{P}\right)$ is a maximal ideal of $R_{P}$, $N_{P}=\left\{\left(\frac{x}{s}, \frac{y}{t}\right) \in F_{P}: s a y-t b x \in P\right\}$ by Theorem 2.6. Let $\varphi: F \longrightarrow F_{P}$, be the natural homomorphism. Then we have $\varphi^{-1}\left(N_{P}\right)=\left\{(x, y) \in F:\left(\frac{x}{1}, \frac{y}{1}\right) \in\right.$ $\left.N_{P}\right\}=\{(x, y) \in F: a y-b x \in P\}=\{(x, y) \in F: r(x, y) \in N$ for some $r \in R \backslash P\}=K(N, P)$.

(2) Suppose that $N$ is a prime submodule and $\left(\frac{1}{1}, \frac{0}{1}\right) \in N_{P}$. Then $\frac{(1,0)}{1}=\frac{(x, y)}{s}$ for some $(x, y) \in N$ and $s \in R \backslash P$. We have $u(s(1,0)-(x, y))=0$ for some $u \in R \backslash P$. Since $u s(1,0) \in N$ and $(1,0) \notin N$, we get that $u s \in P$, a contradiction. Thus $\left(\frac{1}{1}, \frac{0}{1}\right) \notin$ $N_{P}$. Similarly $\left(\frac{0}{1}, \frac{1}{1}\right) \notin N_{P}$. By $(1), K(N, P)=\{(x, y) \in F: a y-b x \in P\}=N$ as $K(N, P)$ is the smallest $P$-prime submodule containing $N$.

Conversely, it can be easily seen that $\{(x, y) \in F: a y-b x \in P\}$ is a $P$-prime submodule of $F$. 
To sum up our results about prime submodules of $F$, combining Corollary 2.3 and Corollary 2.7, we give the following theorem which characterizes all prime submodules of $F$.

Theorem 2.8. Let $N$ be a submodule of $F$.

(1) Assume that $N$ contains $(1,0)$ or $(0,1)$. Then, $N$ is a prime submodule of $F$ if and only if $(N: F)=P$ is a prime ideal of $R$ and $N=R \oplus P$ or $N=P \oplus R$.

(2) Assume that $N$ does not contain $(1,0)$ and $(0,1)$. Then $N$ is a prime submodule of $F$ if and only if $(N: F)=P$ is a prime ideal of $R$ and $N=P \oplus P$ or $N=\{(x, y) \in F: a y-b x \in P\}$, where $(a, b) \in N$ with $R a+R b \nsubseteq P$.

In the following theorem, we determine whether $N$ is a prime submodule of $F$ or not, by using primeness of a certain ideal of $R$. This theorem is a generalization of [ 9 , Proposition 3.4] and a useful primeness test for a finitely generated submodule of $F$.

Theorem 2.9. Let $N$ be an $n$-generated submodule of $F$ with $R=R a_{n}+R b_{n}$. Then $N$ is a prime submodule of $F$ if and only if $\sum_{i=1}^{n-1} R \Delta_{n i}$ is a prime ideal of $R$.

Proof. By the hypothesis, there exist elements $s_{1}, s_{2} \in R$ such that $1=$ $s_{1} a_{n}+s_{2} b_{n}$. Let $L=R\left(a_{n}, b_{n}\right)$ and $L^{\prime}=\left\{(x, y) \in F: s_{1} x+s_{2} y=0\right\}$. Consider the functions $\Psi: R \rightarrow F$ defined by $\Psi(r)=r\left(a_{n}, b_{n}\right)$ and $\Phi: F \rightarrow R$ defined by $\Phi\left(\left(r_{1}, r_{2}\right)\right)=s_{1} r_{1}+s_{2} r_{2}$. Then since $\Phi$ is onto and $R$ is projective, we get that $F=\operatorname{Im} \Psi \oplus \operatorname{ker} \Phi=L \oplus L^{\prime}$. On the other hand by the modularity law, we have $N=L \oplus\left(N \cap L^{\prime}\right)$. Set $c_{i}=s_{1} a_{i}+s_{2} b_{i}(1 \leq i \leq n-1)$. Then $\sum_{i=1}^{n-1} R\left(\left(a_{i}, b_{i}\right)-\right.$ $\left.c_{i}\left(a_{n}, b_{n}\right)\right) \subseteq N \cap L^{\prime}$.

To show that $N=\left(\sum_{i=1}^{n-1} R\left(\left(a_{i}, b_{i}\right)-c_{i}\left(a_{n}, b_{n}\right)\right)\right) \oplus L$, take $(x, y) \in N$. Then $(x, y)=\sum_{i=1}^{n} r_{i}\left(a_{i}, b_{i}\right)$ for some $r_{i} \in R$ and so

$$
\begin{aligned}
(x, y)= & \sum_{i=1}^{n} r_{i}\left(a_{i}, b_{i}\right)-\sum_{i=1}^{n-1} r_{i} c_{i}\left(a_{n}, b_{n}\right) \\
& +\sum_{i=1}^{n-1} r_{i} c_{i}\left(a_{n}, b_{n}\right) \in\left(\sum_{i=1}^{n-1} R\left(\left(a_{i}, b_{i}\right)-c_{i}\left(a_{n}, b_{n}\right)\right)\right) \oplus L
\end{aligned}
$$

and so $N=\left(\sum_{i=1}^{n-1} R\left(\left(a_{i}, b_{i}\right)-c_{i}\left(a_{n}, b_{n}\right)\right)\right) \oplus L$. Therefore we get the equality $N \cap L^{\prime}=\sum_{i=1}^{n-1} R\left(\left(a_{i}, b_{i}\right)-c_{i}\left(a_{n}, b_{n}\right)\right)$.

Now we show that $F=L+R\left(-s_{2}, s_{1}\right)$. We have that

$$
\begin{aligned}
& (1,0)=s_{1}\left(a_{n}, b_{n}\right)+\left(-b_{n}\right)\left(-s_{2}, s_{1}\right) \\
& (0,1)=s_{2}\left(a_{n}, b_{n}\right)+a_{n}\left(-s_{2}, s_{1}\right)
\end{aligned}
$$

These imply that $F=L+R\left(-s_{2}, s_{1}\right)$. Then since $R\left(-s_{2}, s_{1}\right) \subseteq L^{\prime}$ and by the modularity law, it follows that $L^{\prime}=R\left(-s_{2}, s_{1}\right)+\left(L \cap L^{\prime}\right)=R\left(-s_{2}, s_{1}\right)$. Note that 


$$
\begin{aligned}
-s_{2} \Delta_{n i} & =-s_{2}\left(a_{n} b_{i}-b_{n} a_{i}\right)=-s_{2} a_{n} b_{i}+s_{2} b_{n} a_{i} \\
& =-s_{2} a_{n} b_{i}+\left(1-s_{1} a_{n}\right) a_{i}=a_{i}-a_{n}\left(s_{1} a_{i}+s_{2} b_{i}\right) \\
& =a_{i}-c_{i} a_{n},
\end{aligned}
$$

and similarly, we get that $s_{1} \Delta_{n i}=b_{i}-c_{i} b_{n}$.

Then $\left(a_{i}, b_{i}\right)-c_{i}\left(a_{n}, b_{n}\right)=\left(a_{i}-c_{i} a_{n}, b_{i}-c_{i} b_{n}\right)=\Delta_{n i}\left(-s_{2}, s_{1}\right)(1 \leq i \leq n-1)$.

Let $I=\sum_{i=1}^{n-1} R \Delta_{n i}$. Then $N \cap L^{\prime}=I\left(-s_{2}, s_{1}\right)$. Now since $F=L \oplus L^{\prime}$ and $N=L \oplus\left(N \cap L^{\prime}\right)$, it follows that

$$
F / N \simeq L^{\prime} /\left(N \cap L^{\prime}\right)=R\left(-s_{2}, s_{1}\right) / I\left(-s_{2}, s_{1}\right)
$$

On the other hand, if $r \in R$ and $r\left(-s_{2}, s_{1}\right)=(0,0)$, then $r s_{2}=r s_{1}=0$ and hence $r$ $=r 1=\left(r s_{1}\right) a_{n}+\left(r s_{2}\right) b_{n}=0$. Thus we get that $F / N \simeq R / I=R / \sum_{i=1}^{n-1} R \Delta_{n i}$. Thus $N$ is a prime submodule of $F$ if and only if $\sum_{i=1}^{n-1} R \Delta_{n i}$ is a prime ideal of $R$.

Corollary 2.10. Let $R$ be a domain and $a, b \in R$ such that $R a+R b=R$. Then $N=R(a, b)$ is a prime submodule of $F$.

Now we determine a primary decomposition of $N$ when $R$ is a domain.

Lemma 2.11. Let $Q$ be a P-primary ideal of $R$ containing $\mathcal{L}$ and $T_{Q}=\left\{\left(m_{1}, m_{2}\right) \in\right.$ $F: a_{i} m_{2}-b_{i} m_{1} \in Q$ for all $\left.i \in \Lambda\right\}$. Then $T_{Q}=F$ or $T_{Q}$ is a P-primary submodule of $F$ containing $N$.

Proof. If $a_{i}, b_{i} \in Q$ for all $i \in \Lambda$, then $T_{Q}=F$. Suppose that $a_{j} \notin Q$ for some $j \in \Lambda$. Now we prove that $T_{Q}$ is a $P$-primary submodule of $F$.

Let $r \in \sqrt{\left(T_{Q}: F\right)}$. Then $r^{n}(0,1) \in T_{Q}$ for some $n \in \mathbb{Z}^{+}$and so $r^{n} a_{j} \in Q$. Since $a_{j} \notin Q$, we have $r \in P$. Hence $\sqrt{\left(T_{Q}: F\right)} \subseteq P$. Let $r \in P$. $r^{n} \in Q$ for some $n \in \mathbb{Z}^{+}$. It follows that $r^{n}(x, y) \in T_{Q}$ for all $(x, y) \in F$. Therefore $\sqrt{\left(T_{Q}: F\right)}=P$.

Assume that $r m \in T_{Q}$ for $r \in R-P$ and $m=\left(m_{1}, m_{2}\right) \in F$. Then $r\left(a_{i} m_{2}-\right.$ $\left.b_{i} m_{1}\right) \in Q$ for all $i \in \Lambda$. Since $r \notin P$, we get that $m \in T_{Q}$. Thus $T_{Q}$ is a $P$-primary submodule of $F$. Since $\mathcal{L} \subseteq \mathcal{Q}$ we have $N \subseteq T_{Q}$.

Theorem 2.12. Let $R$ be a domain, $N$ be a proper submodule of $F$ with $|\Lambda| \geq 2$ and let $\mathcal{L}$ be a non-zero ideal of $R$ such that $\mathcal{L}=R \Delta_{k l}$ for some $k, l \in \Lambda$. Let $\mathcal{L}=\cap_{i=1}^{n} Q_{i}$ be a minimal primary decomposition of $\mathcal{L}$ with $A s s(\mathcal{L})=\left\{P_{i}\right\}_{i=1}^{n}$. Then,

(a) $\cap_{i=1}^{n} T_{Q_{i}}$ is a primary decomposition of $N$.

(b) If $\left\{a_{j}: j \in \Lambda\right\} \cup\left\{b_{j}: j \in \Lambda\right\} \nsubseteq P_{i}$ for all $1 \leq i \leq n$, then $\cap_{i=1}^{n} T_{Q_{i}}$ is a minimal primary decomposition of $N$ with $\operatorname{Ass}(N)=\left\{P_{i}\right\}_{i=1}^{n}$

(c) If $\mathcal{L}$ has no embedded prime ideal, then $\cap_{i=1}^{n} T_{Q_{i}}$ is a minimal primary decomposition of $N$ with $\operatorname{Ass}(N)=\left\{P_{i}\right\}_{i=1}^{n}$. 
Proof.

(a) Since $N \subseteq T_{Q_{i}}$ for all $i \in\{1, . ., n\}$, we have $N \subseteq \cap_{i=1}^{n} T_{Q_{i}}$. Take an element $(x, y) \in \cap_{i=1}^{n} T_{Q_{i}}$. Then $a_{j} y-b_{j} x \in Q_{i}$ for all $j \in \Lambda$ and $i \in\{1, \ldots, n\}$ and so $a_{j} y-b_{j} x \in \cap_{i=1}^{n} Q_{i}=\mathcal{L}=R \Delta_{k l}$. In particular, there exist $t_{1}, t_{2} \in R$ such that $a_{k} y-b_{k} x=t_{1} \Delta_{k l}$ and $a_{l} y-b_{l} x=t_{2} \Delta_{k l}$. It is easily seen that $(x, y)=$ $\left(a_{k}, b_{k}\right)\left(-t_{2}\right)+\left(a_{l}, b_{l}\right) t_{1} \in N$. Hence $N=\cap_{i=1}^{n} T_{Q_{i}}$. Let $S=\{s \in\{1, \ldots, n\}$ : $a_{i} \notin P_{s}$ for some $i \in \Lambda$ or $b_{j} \notin P_{s}$ for some $\left.j \in \Lambda\right\}$ and $i \in\{1, \ldots, n\}-S$. Then $N \subseteq Q_{i} \oplus Q_{i}$ and $T_{Q_{i}}=F$. Therefore, $N=\cap_{s \in S} T_{Q_{s}} \subseteq \cap_{i \notin S}\left(Q_{i} \oplus Q_{i}\right)$. Then we get that $\cap_{s \in S} Q_{s} \subseteq\left(\cap_{s \in S} T_{Q_{s}}: F\right) \subseteq \cap_{i \notin S}\left(Q_{i} \oplus Q_{i}: F\right)=\cap_{i \notin S} Q_{i}$. It follows that $\cap_{i=1, i \neq j}^{n} Q_{i} \subseteq Q_{j}$ for every $j \notin S$, a contradiction. Thus $S=$ $\{1, \ldots, n\}$ and so $N=\cap_{i=1}^{n} T_{Q_{i}}$ is a primary decomposition of $N$.

(b) Suppose that $\cap_{i=1, i \neq j}^{n} T_{Q_{i}} \subseteq T_{Q_{j}}$ for some $1 \leq j \leq n$. Take an element $r \in$ $\cap_{i=1, i \neq j}^{n} Q_{i}-Q_{j}$. Then $(0, r) \in \cap_{i=1, i \neq j}^{n} T_{Q_{i}}$. We can assume that $a_{t} \notin P_{j}$ for some $t \in \Lambda$. Since $(0, r) \in T_{Q_{j}}$ we have $\left(-r a_{t}\right) \in Q_{j}$ and so $r \in Q_{j}$. But this is a contradiction. Thus $\cap_{i=1, i \neq j}^{n} T_{Q_{i}} \not T_{Q_{j}}$ for all $1 \leq j \leq n$. So $\cap_{i=1}^{n} T_{Q_{i}}$ is a minimal primary decomposition of $N$ with $\operatorname{Ass}(N)=\left\{P_{i}\right\}_{i=1}^{n}$.

(c) Suppose that $\cap_{i=1, i \neq j}^{n} T_{Q_{i}} \subseteq T_{Q_{j}}$ for some $1 \leq j \leq n$. Then $\sqrt{\left(\cap_{i=1, i \neq j}^{n} T_{Q_{i}}: F\right)}$ $\subseteq \sqrt{\left(T_{Q_{j}}: F\right)}$ and so $\cap_{i=1, i \neq j}^{n} P_{i} \subseteq P_{j}$. It follows that $P_{i} \subseteq P_{j}$ for some $1 \leq i \leq n, i \neq j$. Since $\mathcal{L}$ has no embedded prime, we get that $P_{i}=P_{j}$, a contradiction.

Note that the first condition on $\mathcal{L}$ in Theorem 2.12 is satisfied if $N$ is two-generated or $N$ is finitely generated and $R$ is a valuation domain.

Corollary 2.13. Let $R$ be a domain, $N$ be a proper submodule of $F$ with $|\Lambda| \geq 2$ and let $\mathcal{L}$ be a non-zero ideal of $R$ such that $\mathcal{L}=R \Delta_{k l}$ for some $k, l \in \Lambda$. If $\mathcal{L}$ has the unique prime ideal factorization $P_{1}^{t_{1}} \ldots P_{n}^{t_{n}}$ with distinct maximal ideals $P_{i},(1 \leq i \leq n)$, then $\cap_{i=1}^{n} T_{P_{i}^{t_{i}}}$ is a minimal primary decomposition of $N$ with $\operatorname{Ass}(N)=\left\{P_{i}\right\}_{i=1}^{n}$.

Proof. $\mathcal{L}=P_{1}^{t_{1}} \cap \ldots \cap P_{n}^{t_{n}}$ is a minimal primary decomposition of $\mathcal{L}$ with $A s s(\mathcal{L})=\left\{P_{i}\right\}_{i=1}^{n}$. Suppose that there exists an $i \in\{1, \ldots, n\}$ such that $a_{j}, b_{j} \in P_{i}^{t_{i}}$ for all $j \in \Lambda$. Then we get that $\mathcal{L}=P_{1}^{t_{1}} \ldots P_{i}^{2 t_{i}} \ldots P_{n}^{t_{n}}$. But this contradicts with the unique prime ideal factorization of $\mathcal{L}$. So $\cap_{i=1}^{n} T_{P_{i}^{t_{i}}}$ is a minimal primary decomposition of $\mathcal{L}$ by Theorem 2.12-(b).

Finally we give two examples as applications of our results for free modules with two generators over domains which are not principal ideal domains.

Example 2.14. Let $R$ be the polynomial ring $\mathbb{Z}[X]$ and $N$ the submodule $R(X-$ $2, X-2)+R(1, X)$. Then $\mathcal{L}=R \Delta_{12}=R(X-2)(X-1)$ and $R(X-2) \cap R(X-$ 
1 ) is a minimal primary decomposition of $\mathcal{L}$. By applying Theorem 2.12-(b), we get that $N=T_{R(X-2)} \cap T_{R(X-1)}$ is a minimal primary decomposition of $N$, where $T_{R(X-2)}=\{(f, g) \in F: X f-g \in R(X-2)\}$ and $T_{R(X-1)}=\{(f, g) \in F: f-g$, $X f-g \in R(X-1)\}$.

Example 2.15. Let $R=\mathbb{Z}[\sqrt{-5}]$ and $N=R(1+\sqrt{-5}, 3)+R(1,1-\sqrt{-5})$. Then $\mathcal{L}=R 3$. It is well-known that $R$ is a Dedekind domain and the unique prime ideal factorization of $\mathcal{L}$ is $P_{1} P_{2}$, where $P_{1}=R 3+R(1+\sqrt{-5}), P_{2}=R 3+R(1-\sqrt{-5})$. By applying Corollary 2.13, we get that $N=T_{P_{1}} \cap T_{P_{2}}$ is a minimal primary decomposition of $N$, where $T_{P_{1}}=\left\{(x, y) \in F: y-x(1-\sqrt{-5}) \in P_{1}\right\}$ and $T_{P_{2}}=R \oplus P_{2}$.

\section{ACKNOWLEDGMENT}

The first author thanks the Scientific Technological Research Council of Turkey (TUBITAK) for the financial support.

The authors were supported by the Scientific Research Project Administration of Akdeniz University.

\section{REFERENCES}

1. M. Alkan and B. Saraç, On Primary Decompositions and Radicals of Submodules, Proc. Jangjeon Math. Soc., 10(1) (2007), 71-81.

2. S. Çeken and M. Alkan, On Radical Formula over Free Modules with Two Generators, Book Series: AIP Conference Proceedings, Vol. 1389-1, 2011, pp. 333-336.

3. S. Hedayat and R. Nekooei, Characterization of Prime Submodules of a Finitely Generated Free Module Over a PID, Houston J. Math., 31(1) (2005), 75-85.

4. S. Hedayat and R. Nekooei, Prime and Radical Submodules of Free Modules Over a PID, Houston J. Math., 32(2) (2006), 355-367.

5. S. Hedayat and R. Nekooei, Primary Decomposition of Submodules of a Finitely Generated Module Over a PID, Houston J. Math., 32(2) (2006), 369-377.

6. C. P. Lu, Prime Submodules of Modules, Comment. Math. Univ. Sancti Paulli, 33(1) (1984), 61-69.

7. R. L. McCasland and M. E. Moore, Prime Submodules, Comm. Algebra, 20(6) (1992), 1803-1817.

8. D. Pusat-Yllmaz and P. F. Smith, Radicals of Submodules of Free Modules, Comm. in Alg., 27(5) (1999), 2253-2266.

9. D. Pusat-Yllmaz, On Prime Submodules of Finitely Generated Free Modules, Turk. J. Math., 27 (2003), 329-342.

10. Y. Tıraş and A. Harmancl, On Prime Submodules and Primary Decomposition, Czech. Math. J., 50(125) (2000), 83-90. 
11. Y. Tiraş, A. Harmancl and P. F. Smith, A Characterization of Prime Submodules, $J$. Algebra, 212(2) (1999), 743-752.

Seçil Çeken and Mustafa Alkan

Akdeniz University

Department of Mathematics

Antalya, Turkey

E-mail: secilceken@akdeniz.edu.tr alkan@akdeniz.edu.tr 